\title{
Interventions infirmières pour promouvoir la fidélisation des donneurs de sang: une revue rapide et réaliste de la littérature
}

\author{
Nursing interventions to promote blood donation commitment: \\ A rapid and realistic literature review
}

Cécile Marie DUPIN, Infirmière, Ph.D, Professeure assistante, Haute école de santé, Genève, Suisse

Sarah DEUBELBEISS, Infirmière, Haute école de santé, Genève, Suisse

Katy DOS SANTOS RODRIGUES, Infirmière, Haute école de santé, Genève, Suisse

Daycielly MORAIS DE OLIVEIRA, Infirmière, Haute école de santé, Genève, Suisse

Camille THENTZ, Infirmière assistante, M.Sc, Haute école de santé, Genève, Suisse

Muriel QUILLEAU, Infirmière, Centre de transfusion des hôpitaux universitaires de Genève, Suisse

\begin{abstract}
RÉSUMÉ
Contexte : la transfusion sanguine sauve des vies. Le don du sang est un facteur clef dans les systèmes de santé. Cependant, la pénurie subsiste. Pour garantir des réserves suffisantes en sang, les services de santé ont besoin de la collaboration de donneurs volontaires. Les infirmières des centres de transfusion sont des acteurs clefs de fidélisation. Les motivations des donneurs ainsi que les interventions infirmières promouvant la fidélisation doivent être recensées et analysées.

Objectif : identifier les mécanismes existants chez les donneurs pouvant être les supports d'interventions infirmières.

Méthode : une revue réaliste de la littérature telle que définie par Pawson a été conduite en 2018. Les recherches d'articles ont été effectuées dans deux bases de données. Cette stratégie a permis d'identifier neuf articles scientifiques. Ils ont été analysés selon le cadre réaliste.

Résultats : l'analyse a mis en évidence trois pistes d'actions : les mécanismes issus des expériences individuelles des donneurs, les interventions existantes qui permettent aux donneurs de dépasser l'intention pour concrétiser un don, enfin les interventions interactives dans la dyade entre infirmière et donneur. La relation doit favoriser une bonne expérience du don, notamment chez les nouveaux donneurs pour pouvoir influencer positivement la fidélisation.
\end{abstract}

Mots clés : don du sang, donneur de sang, interventions infirmières, motivation.

Pour cifer l'article :

Dupin CM, Deubelbeiss S, Dos Santos Rodrigues K, Morais De Oliveira D, Thentz C, Quilleau M, et al. Interventions infirmières pour promouvoir la fidélisation des donneurs de sang : une revue rapide et réaliste de la littérature. Rech Soins Infirm. 2019 Mar;(136):16-27.

Adresse de correspondance :

Cécile Marie Dupin : cecile.dupin@hesge.ch 


\begin{abstract}
Background: Blood transfusions save lives. Blood donation is a key factor in health systems. However, there is a long-term shortage of blood. To ensure sufficient blood supply, health services need to collaborate with voluntary donors. Nurses who work at transfusion centers are key actors in encouraging blood donor commitment. The motivations of the donors as well as effective nursing interventions should be identified and analyzed.

Objective: To identify existing nursing interventions that encourage blood donation and favor collaborative relationships between nurses and donors.

Method: A realistic review of the literature as defined by Pawson was conducted in 2018. The articles were selected from two scientific databases. This strategy identified nine scientific articles, which were analyzed using the realistic framework.

Results: The analysis highlighted three domains of intervention: the mechanisms stemming from the individual experiences of donors; the existing interventions that allow donors to go from intending to give blood to actually giving blood; and finally the interactive interventions in the dyad between nurses and donors. The relationship, especially with new donors, should positively influence donation and commitment.
\end{abstract}

Keywords: Blood donation, blood donor, commitment, nursing interventions.

\title{
NOTE DES AUTEURS
}

Ce travail a été réalisé dans le cadre d'une formation en soins infirmiers à la Haute école de santé de Genève en vue de l'obtention du titre de Bachelor of Science HES-SO en Soins infirmiers.

\section{LE BESOIN EN SANG ET LA PROMOTION DU DON DU SANG VOLONTAIRE}

Selon l'Organisation mondiale de la santé (OMS) (1), la politique nationale de transfusion sanguine et l'organisation des services pour un approvisionnement en sang suffisant doit faire partie intégrante de l'infrastructure sanitaire d'un pays. Chaque année dans le monde 112,5 millions de dons de sang sont collectés. L'approvisionnement en sang est différent selon les pays : le taux médian de dons du sang dans les pays à revenu élevé est de 32,1 pour 1000 habitants, contre 14,9 dans les pays à revenu intermédiaire supérieur, 7,8 dans les pays à revenu intermédiaire inférieur et 4,6 dans les pays à faible revenu.

La transfusion sanguine contribue à sauver des vies lors de situations pouvant compromettre le pronostic vital des patients ${ }^{1}$. Elle peut aider les patients souffrant d'une pathologie mortelle ou ayant subi des traumatismes graves à la suite d'un accident ou lors d'hémorragies sévères, par exemple suite à un accouchement ou dans le cas de catastrophes naturelles. L'accès au sang reste une difficulté et la pénurie est mondiale et constante $(2,3)$. En effet, le nombre de dons reçus est insuffisant pour couvrir l'intégralité des

\footnotetext{
${ }^{1}$ Dans cet article, le mot patient sera utilisé à titre épicène, il inclura les patients et les patientes.
}

besoins (4). Chaque année dans plusieurs pays, les besoins en sang sont supérieurs à l'offre. Selon l'OMS (1), il existe trois types de donneurs de sang : les donneurs volontaires non rémunérés, les donneurs pour un membre de la famille, les donneurs rémunérés. Selon le Dr Margaret Chan, le meilleur moyen de garantir un approvisionnement sûr et suffisant en sang ou produits sanguins pour la transfusion est de disposer d'un bon approvisionnement basé sur les dons réguliers de sang de donneurs volontaires et non rémunérés (4). Ce groupe de donneurs se trouverait être le plus fiable, car il participe à ce don de manière altruiste. Un donneur de sang volontaire non rémunéré donne librement du sang, du plasma ou des éléments figurés du sang. II s'engage à ne recevoir aucun paiement, que ce soit en espèces ou en nature, qui puisse être considéré comme un substitut à l'argent (4). Ces donneurs font donc partie intégrante du système de santé. Les résultats communiqués par l'OMS entre 2008 et 2013 montrent une augmentation de 10,7 millions de donneurs volontaires non rémunérés au sein de 159 pays.

En 2011, l'OMS ainsi que la fédération internationale des Sociétés de la Croix-Rouge et du Croissant-Rouge ont élaboré un objectif commun visant à l'obtention de $100 \%$ de dons de sang volontaires et non rémunérés dans le monde entier, permettant d'assurer un approvisionnement fiable et durable en sang (4). Un constat international insiste sur le besoin de recruter ces nouveaux donneurs de sang, de fidéliser les donneurs réguliers $(2,5,8)$ et de cibler et communiquer auprès des jeunes $(3,8,9)$ 


\section{MÉCANISMES ET FREINS AU DON DU SANG}

Les donneurs les plus réguliers sont les hommes, les 18-35 ans, les personnes vivant en couple, ainsi que les étudiants et les employés $(2,10)$. La plupart des donneurs sont motivés par la croyance que peut-être un jour eux-mêmes ou leurs proches auront besoin de sang. Les facteurs facilitateurs connus sont un accès au centre de don facile $(2,7)$, la volonté d'aider les autres, notamment par empathie $(3,10)$, ou encore avoir un proche ayant eu besoin d'une transfusion récente. Certaines stratégies de fidélisation existantes sont présentées dans le tableau 1.

Certains facteurs empêchent la concrétisation du premier don, par méconnaissance du processus de don du sang chez les non donneurs (9). Pour certains donneurs, il est difficile, voire impossible, de pouvoir se libérer de leurs tâches quotidiennes (vie de famille, activités personnelles et professionnelles). Par ailleurs, certains estiment que le temps de déplacement jusqu'au point de collecte, la durée de la prise en charge ainsi que le moment de repos

Mettre en avant la convivialité de ce moment ainsi que l'échange avec les donneurs réguliers rendant cette expérience plus agréable et plus positive à leurs yeux.

Laisser en mémoire une expérience positive qui pourrait permettre la fidélisation régulière de certains donneurs.

Possibilité d'agir à différents niveaux tel que « le marketing relationnel et le marketing expérientiel » : développer des stratégies de communication en mettant en avant les bénéfices de ces dons envers la société, à travers de grandes affiches publicitaires exposées dans les rues ainsi que des flyers mis à disposition dans différents lieux.

Le “bouche-à-oreille » de la part des donneurs fidèles : un moyen communicatif et interactif permettant de partager en parlant de leur expérience, des lieux, des horaires de collecte et de l'importance de donner son sang.

Pour favoriser le retour ponctuel des donneurs, mettre en place la communication personnelle (email, sms, courrier).

Fixer une date avec le Centre de Transfusion Sanguine pour un prochain don, afin d'engager la fidélisation et de ce fait permettre une meilleure gestion du temps personnel.

Travailler sur les croyances et rassurer, ce qui permet d'atténuer les craintes et d'attirer de nouveaux donneurs (pp.13-20).

Tableau 1:

Stratégies permettant la fidélisation des donneurs (Ambroise et al., 2010) obligatoire après le don demandent de la disponibilité, donc du temps $(6,11)$. Certaines études relèvent que l'un des freins supplémentaire est la durée qui sépare le premier don du second : grâce à leur premier don, ils ont pu combattre leur peur, mais n'ont pas encore projeté cet acte comme un acte coutumier (9). Avant de pouvoir effectuer le don, celui-ci est déjà représenté comme un acte engageant, de longue durée. Un second frein est l'acte en lui-même, à savoir un prélèvement sanguin avec une aiguille qui est perçu comme une source supplémentaire de stress. Parmi les barrières identifiées se retrouvent les représentations et vécus : peur du sang, des aiguilles, de « se sentir mal » (12).

\section{ORGANISATION DU SYSTÈME DE SANTÉ SUISSE ET CONTEXTE GENEVOIS}

Cerner les raisons et motivations individuelles qui amènent les volontaires à donner leur sang peut permettre de les insérer dans les actions de santé publique. Identifier les populations de donneurs et élaborer des stratégies de communication destinées à les sensibiliser peut maximiser l'impact des journées de don de sang (13). La collaboration entre la CroixRouge suisse et les treize services régionaux de Centre de transfusion sanguine (CTS) assure l'approvisionnement en produits sanguins des hôpitaux (13).

En Suisse, la dose de 770 dons de sang par jour est actuellement requise. $80 \%$ de la population suisse aura besoin de sang au moins une fois dans sa vie. Seulement $5 \%$ de la population donne son sang $(13,14)$. Si le sang n'est pas collecté avec des donneurs volontaires, les structures de santé doivent acheter des poches de sang, ce qui entraîne un coût important. En 2013, à Genève, 18.000 dons ont été effectués ce qui équivaut à environ $2 \%$ à $3 \%$ de la population genevoise (15). Le CTS de Genève s'appuie sur un réseau constitué d'environ 11.000 donneurs réguliers, mais qui ne représente que $3.66 \%$ de la population et seulement $2.45 \%$ de façon régulière en 2016 . De plus, au vu du vieillissement de la population, les besoins de sang pourraient être augmentés à moyen et long terme.

Le CTS des Hôpitaux universitaires de Genève (HUG) s'adresse au grand public selon différentes stratégies et différents modes de communication permettant la collecte de sang tout au long de l'année. Une des premières techniques utilisée est l'envoi d'un courrier postal chez le donneur de manière trimestrielle afin de rappeler le don. Par ailleurs, pour contribuer au recensement de nouveaux donneurs, différentes campagnes publicitaires sont mises en place dans les transports publics ainsi que des événements permettant la sensibilisation du grand public. De plus, l'évolution des réseaux sociaux rend la transmission de l'information plus accessible et rapide. Le CTS suit l'évolution des médias pour accroître le recrutement de nouveaux donneurs (15). 


\section{LE RÔLE INFIRMIER DANS LE DON DU SANG : APPORTS DU MODELE DE NOLA PENDER}

\section{Rôle infirmier dans le don du sang}

Les infirmières jouent un rôle pivot dans le processus du don de sang. Elles sont garantes des évaluations de santé permettant le don, du niveau de soins élevé pour la sécurité du donneur et du futur receveur. Elles permettent que le sang récolté soit de bonne qualité et cohérent avec les normes édictées par les sociétés savantes (16). A Genève où l'entretien s'effectue avec une infirmière, cette dernière est dotée de suffisamment de compétences pour qu'en cas de problèmes, elle en informe le médecin référent du service. Celle-ci possède les connaissances et la capacité d'agir de façon appropriée face aux situations individuelles de chaque donneur (17).

\section{- Présentation du modèle de Pender}

Pour mieux comprendre le rôle de l'infirmière, nous nous référons au modèle de l'infirmière Nola Pender " Health Promotion Model » (18) faisant partie du paradigme de l'intégration et impliquant une prise en soins holistique du patient. Le paradigme de l'intégration a pour signification la reconnaissance des éléments multiples et des manifestations qui se produisent dans le contexte environnemental. L'infirmière considère le patient comme un être bio-psycho-socio-spirituel, un tout. Lorsqu'elle est en situation de collaboration avec le patient, elle prend en compte son expérience de vie, les interactions avec sa famille et son environnement. Le modèle de promotion de la santé de Pender appartient au groupe des théories intermédiaires. Ce modèle a un degré d'abstraction intermédiaire ce qui permet sa mise en application quotidienne dans la pratique infirmière. II permet d'étudier l'interaction entre l'individu et son environnement ainsi que les actions qui l'influencent dans la prise de décisions de maintenir ou d'améliorer son état de santé (19). Le modèle fait partie de l'école d'apprentissage de la santé qui a pour but de maintenir, renforcer et développer la santé du patient en l'engageant activement dans son processus d'apprentissage (20).

\section{- Métaparadigme infirmier selon Pender}

L'ensemble des savoirs disciplinaires infirmiers se basent sur quatre concepts fondamentaux, les métaparadigmes : la santé, le soin infirmier, la personne et l'environnement. Selon Pender, l'individu est perçu comme un être biopsycho-social-spirituel dans un environnement qui évolue. Les caractéristiques individuelles ainsi que les différentes expériences de vie participent et influencent son état de santé (18). Les croyances ainsi que les expériences vécues ont un impact sur la personne, pouvant l'amener ainsi à être sensibilisée par le don du sang. C'est le cas lorsqu'à une étape de sa vie, la personne et/ou un proche est confronté au besoin de sang, ce qui peut être un déterminant clé au fait de devenir donneur de sang. Toujours d'après Pender, le soin est lié à la personne et crée un impact sur l'individu, sa famille ainsi que son entourage. Le soin a pour but de rendre la personne autonome, afin qu'elle parvienne à trouver des moyens favorables à la gestion de sa santé de manière optimale. Concernant le don du sang, le respect des bonnes pratiques en matière de prévention et de soins doit être maîtrisé par l'infirmière. Elle a un rôle clé lors du don du sang, elle intervient au niveau de la pratique du soin, du dépistage et au sujet des éventuels critères pouvant empêcher le bon déroulement du prélèvement du don. En effet, cela exige d'elle une pratique d'experte en soins (17).

Selon Pender, la santé évolue tout au long de la vie et se définit comme l'adaptation des capacités de l'être humain, qu'elles soient intrinsèques ou extrinsèques. Elle se concrétise par des auto-soins appropriés et des relations interpersonnelles satisfaisantes, tout en faisant des ajustements pour préserver l'intégrité de la personne et une certaine osmose avec l'environnement, donc la promotion de la santé (18). Dans le cadre du don du sang, il est constaté des méconnaissances des critères pour devenir un potentiel donneur. Les différentes stratégies de communication mises en place essayent de répondre à cette problématique. Des stratégies de littéracie pourraient avoir un impact sur la population en matière de connaissances, permettant ainsi aux personnes de devenir donneur plus facilement (9). D'après Pender, l'environnement comprend le contexte social (relations interpersonnelles), culturel (religion, croyances et valeurs) et physique (habitation, travail) de la personne. En ce qui concerne le don du sang, les expériences vécues ainsi que les interventions dans l'environnement des donneurs peuvent avoir une influence conséquente sur les motivations au don (21).

\section{Les fondements du modèle}

Le modèle de Pender contient des fondements centraux. Le premier porte sur les " caractéristiques et expériences individuelles » et comprend les expériences vécues et les facteurs personnels. Pender relève que les expériences du passé, les facteurs biologiques (genre, âge, etc.), psychologiques (motivations, estime de soi, etc.) et socioculturels (éducation, religion, etc.) ont une influence sur les comportements de santé (19).

Le deuxième fondement est appelé " comportements spécifiques des connaissances et affects ». II représente les avantages et les obstacles perçus par la personne lors d'un comportement de santé. L'auto-efficacité de la personne lui permet de juger de sa capacité d'organisation et d'exécution d'un comportement. Finalement, ce fondement repose sur les influences interpersonnelles et situationnelles en lien avec les croyances ou les connaissances de la personne, de ses pairs ou de sa famille. Ces différentes perceptions guident la personne à s'interroger lors de l'adoption d'un comportement en matière de santé (18). 
Le troisième fondement dénommé "résultats en comportements » comprend l'influence entre l'intention et l'identification d'une stratégie planifiée, les préférences de la personne et les exigences immédiates. La mise en œuvre de comportements en matière de santé peut être favorisée par un plan d'action adapté au quotidien de la personne. Néanmoins, cette adoption de comportements en matière de santé peut être entravée par des impératifs comme le travail ou les responsabilités familiales (18). Les valeurs socles du modèle de Pender sont présentées dans la tableau 2.

\section{OBJECTIF}

La présente revue de littérature vise à identifier les mécanismes issus des expériences individuelles des donneurs pouvant être les supports de collaboration avec l'infirmière, en se basant sur le modèle infirmier de Nola Pender.

\section{MÉTHODE}

\section{Ancrage réaliste}

L'approche réaliste, ancrée dans la tradition de recherche critique, telle que décrite par Ray Pawson et Nick Tilley $(22,23)$ a pour point de départ la distinction opérée

Les personnes cherchent à trouver des conditions de vie dans lesquelles elles peuvent exprimer leur propre potentiel de santé.

Les personnes ont une capacité de réflexion propre à ellemême, qui leur permet d'identifier leurs propres compétences.

Les personnes évoluent en intégrant différents comportements dans le but d'acquérir une stabilité personnelle.

Les personnes cherchent à contrôler de façon active leur propre comportement.

Les individus dans leur complexité biopsychosociale interagissent avec l'environnement en le transformant progressivement et laissant ainsi l'environnement les transformer eux aussi.

Les professionnels de la santé constituent une partie de l'environnement qui va influencer les personnes au courant de leur vie.

L'initiative prise par soi-même reconfigure les schémas interactifs «personne-environnement » qui sont essentiels pour favoriser le changement de comportement (traduction libre, p.402-3).

Tableau 2 :

Postulats et les valeurs du modèle de Nola Pender (Alligood, 2014) entre l'empirique (ce qui est observé), les événements et expériences qui peuvent/ne peuvent pas être observés et le domaine des mécanismes sociaux sous-jacents. L'explication consiste à déceler les mécanismes sousjacents qui génèrent les comportements. Ray Pawson (23) a détaillé la procédure des revues réalistes de la littérature. II s'agit d'un type de revue qui a pour objectif d'identifier des mécanismes existants. Un mécanisme peut être défini comme : " une entité sous-jacente, des processus ou des structures qui opèrent dans des contextes particuliers pour générer des résultats » (22). Basées sur une théorie ou un modèle existant, ces revues permettent de compléter les théories ou modèles existants, ici le modèle de Pender pour trouver les interventions prometteuses entre infirmières et donneur de sang. Ces revues sont dites " rapides " lorsqu'elles sont un processus de synthèse des connaissances afin de répondre aux problèmes émergents, tout en préservant les éléments fondamentaux de la méthodologie réaliste (24). Ray Pawson (25) a détaillé la procédure de ces revues en 6 étapes :

\section{- Identification de la question de la revue}

La question est la suivante : quelles interventions infirmières envers le donneur peuvent promouvoir la fidélisation au don du sang?

\section{Stratégie de recherche documentaire}

Cette revue de littérature a été réalisée selon l'équation de recherche PICO (Population, Intervention, Comparaison, Outcome). Afin d'obtenir des résultats pertinents, des mots-clés précis issus de la question de recherche ont été sélectionnés. Ils ont été traduits en anglais sur le portail HeTOP (Health Terminology/Ontology Portal). Cette stratégie a permis de révéler les termes suivants : $P$ : Don du sang, donneur de sang (Blood donation, blood donor) ; I : Soins infirmiers (Nursing care, Nursing Interventions), C : (comparaison) non pertinent pour cette question ; $\mathrm{O}$ : Motivation, Fidélisation (Commitment). Ensuite les termes ont été traduits sur le portail de MeSH-Terms correspondant (Medical Subject Headings) pour cibler la base de données PubMed et sur CINAHL HEADINGS pour la base de données CINAHL.

Les mots-clés ont ensuite été entrés dans ces deux bases de données scientifiques: PubMed (spécialisé en sciences biomédicales) et CINAHL (spécialisé en sciences infirmières et paramédicales) afin d'extraire puis de sélectionner les articles pertinents et spécifiques à la question de recherche. Les filtres des 10 dernières années, essais cliniques/MW Word in Subject Heading Full texts 2007-2018/ ont également été utilisés afin de se baser sur des articles récents.

Le diagramme de flux (figure 1) permet de visualiser le processus d'identification des articles scientifiques dans les bases de données. En se basant sur la méthode PICO 


\section{Interventions infirmières pour promouvoir la fidélisation des donneurs de sang : une revue rapide et réaliste de la littérature}

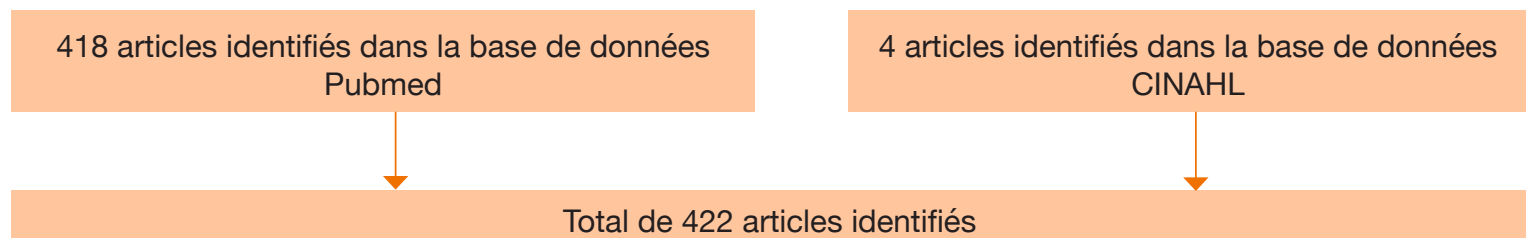

Total de 422 articles identifiés

413 articles exclus sur la base du titre et/ou du résumé

(Etudes non empiriques et/ou non sur le don)

Total de 13 articles éligibles pour l'analyse

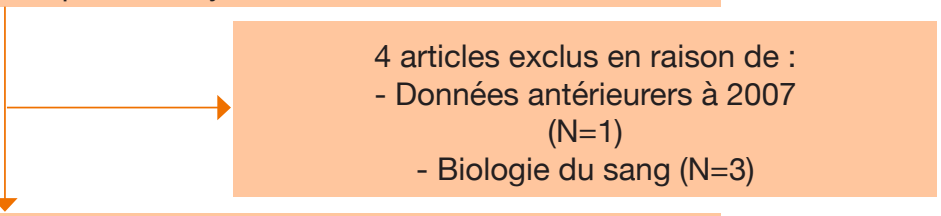

Total de 9 articles inclus dans l'analyse critique réaliste

Figure 1:

Diagramme de flux de sélection des articles

et en l'appliquant à la problématique du travail, des critères d'inclusion (articles en français, portugais ou anglais) et d'exclusion (revues de la littérature) ont été déterminés afin que les articles sélectionnés puissent être pertinents.

\section{Évaluation de la qualité des articles retenus}

Les neuf articles sélectionnés pour cette revue ont été publiés entre 2007 et 2017. Parmi ceux-ci, huit sont des études quantitatives et une est une étude mixte. Selon Polit \& Beck, il y quatre niveaux de preuve, N4 étant le niveau le plus faible (Etudes de cas) et $\mathrm{N} 1$ le plus fort (Etudes interventionnelles contrôlées) (26). Parmi les études quantitatives qui ont été sélectionnées, deux sont de niveau N1, une est de niveau N2, cinq sont de niveau N3 et une de niveau N4. Parmi les études sélectionnées, trois ont été effectuées au Canada, deux en Europe (en Italie et au Pays-Bas), une en Chine, une au Brésil, une en Ethiopie et une en Australie. Parmi les articles inclus dans cette revue, quatre ont appliqué la Théorie du comportement planifié d'Azjen (TCP) (27) comme cadre théorique $(6,21,28,29)$.

L'étape quatre d'extraction des données a été réalisée d'abord sur fiches de lectures systématiques précisant contexte de réalisation de l'étude, objet de l'article et cadre théorique, méthodologie de la recherche, présentation des résultats, éthique, apports de l'article, limites (méthodologiques ou de connaissances), pistes de réflexion. Ensuite des tableaux comparatifs des articles présentaient de manière synthétique pour chaque article : auteurs-année, population et type étude, but de l'étude, intervention, méthode et/ou instrument de mesure, principaux résultats, limites. Les résultats ci-dessous montrent la synthèse des données (étape cinq). Le présent article permet la sixième et dernière étape de la revue réaliste : dissémination des résultats (25).

\section{RÉSULTATS}

L'analyse et la comparaison des neuf articles ont mis en évidence trois types d'actions essentielles : celles basées sur des expériences individuelles des donneurs, les interventions qui permettent de dépasser l'intention pour aboutir à concrétiser un don, enfin les interventions interactives qui peuvent être mobilisées lors de la rencontre entre infirmière et donneurs (tableau 3).

\section{Les actions issues des expériences individuelles}

Parmi les 859 participants à une étude quantitative descriptive simple par questionnaire d'auto-évaluation (10) qui décrit le nombre annuel et total de dons et l'engagement des donneurs envers les organisations, $56 \%$ des participants ont comme principale motivation, de venir en aide à l'autre, souvent influencés par la famille ou les proches. Le don est perçu comme un geste altruiste. Plus précisément, en réponse à la question " pourquoi avez-vous choisi de devenir un donneur de sang ? ", les personnes âgées entre 18-35 ans ont déclaré être généralement influencées par leur famille ; les femmes ont déclaré qu'elles donnent leur sang par choix personnel et qu'elles sont également influencées par leur partenaire, alors que les hommes ont déclaré être plutôt influencés par des amis. 


\begin{tabular}{|c|c|c|c|c|c|c|c|c|c|c|}
\hline $\begin{array}{l}\text { Catégories de } \\
\text { mécanismes }\end{array}$ & $\begin{array}{l}\text { Données en lien avec le } \\
\text { modèle de Nola Pender }\end{array}$ & $\begin{array}{l}\text { Wevers } \\
\text { et al., } \\
2014 \\
(\mathrm{~N} 1)\end{array}$ & $\begin{array}{l}\text { Araujo } \\
\text { et al., } \\
2010 \\
\text { (N 2) }\end{array}$ & $\begin{array}{l}\text { Godin } \\
\text { et al., } \\
2007 \\
\text { (N 3) }\end{array}$ & $\begin{array}{l}\text { Bani } \\
\text { et al., } \\
2011 \\
\text { (N 3) }\end{array}$ & $\begin{array}{l}\text { Godin } \\
\text { et al., } \\
2012 \\
\text { (N 3) }\end{array}$ & $\begin{array}{l}\text { Balegh } \\
\text { et al., } \\
2015 \\
\text { (N 3) }\end{array}$ & $\begin{array}{l}\text { Arage } \\
\text { et al., } \\
2015 \\
\text { (N 3) }\end{array}$ & $\begin{array}{l}\text { Hyde } \\
\text { et al., } \\
2012 \\
\text { (N 4) }\end{array}$ & $\begin{array}{l}\text { Chen, } \\
2017 \\
\text { (N4) }\end{array}$ \\
\hline \multirow[t]{4}{*}{$\begin{array}{l}\text { Mécanismes } \\
\text { du don }\end{array}$} & $\begin{array}{l}\text { « caractéristiques } \\
\text { et expériences } \\
\text { individuelles » }\end{array}$ & $\sqrt{ }$ & & $\sqrt{ }$ & $\sqrt{ }$ & $\sqrt{ }$ & $\sqrt{ }$ & $\sqrt{ }$ & $\sqrt{ }$ & $\sqrt{ }$ \\
\hline & Les vécus de dons & $\sqrt{ }$ & & $\sqrt{ }$ & $\sqrt{ }$ & $\sqrt{ }$ & $\sqrt{ }$ & & & \\
\hline & L'altruisme & $\sqrt{ }$ & $\sqrt{ }$ & & $\sqrt{ }$ & & & $\sqrt{ }$ & & $\sqrt{ }$ \\
\hline & $\begin{array}{l}\text { La fidélisation des } \\
\text { donneurs }\end{array}$ & & & $\sqrt{ }$ & & & $\sqrt{ }$ & & $\sqrt{ }$ & $\sqrt{ }$ \\
\hline \multirow[t]{5}{*}{$\begin{array}{l}\text { Leviers des } \\
\text { interventions }\end{array}$} & «Auto-efficacité » & $\sqrt{ }$ & & & $\sqrt{ }$ & $\sqrt{ }$ & & $\sqrt{ }$ & & \\
\hline & $\begin{array}{l}\text { Agir sur les contraintes } \\
\text { de temps }\end{array}$ & $\sqrt{ }$ & & & & & & & & \\
\hline & $\begin{array}{l}\text { Comprendre le vécu } \\
\text { d'être exclus pour des } \\
\text { raisons médicales }\end{array}$ & $\sqrt{ }$ & & & & & & & & \\
\hline & $\begin{array}{l}\text { Investissement des } \\
\text { soignants lors de la } \\
\text { prise en soin }\end{array}$ & & $\sqrt{ }$ & & & & & & & \\
\hline & $\begin{array}{l}\text { Echanger sur les } \\
\text { craintes/peurs du } \\
\text { monde médical } \\
\end{array}$ & $\sqrt{ }$ & & & & & & & & \\
\hline \multirow{3}{*}{$\begin{array}{l}\text { Mécanismes } \\
\text { de } \\
\text { collaboration } \\
\text { soignant- } \\
\text { donneur }\end{array}$} & $\begin{array}{l}\text { "La mise en œuvre de } \\
\text { comportements " }\end{array}$ & & $\sqrt{ }$ & & $\sqrt{ }$ & & & $\sqrt{ }$ & & \\
\hline & $\begin{array}{l}\text { Les similitudes entre } \\
\text { professionnels de la } \\
\text { santé et grand public }\end{array}$ & & & & $\sqrt{ }$ & & & $\sqrt{ }$ & & \\
\hline & $\begin{array}{l}\text { L'impact de l'attitude } \\
\text { des professionnels de } \\
\text { la santé sur le donneur }\end{array}$ & & $\sqrt{ }$ & & & & & & & \\
\hline
\end{tabular}

Tableau 3 :

Tableau synoptique des résultats au regard de la théorie de Pender

Ensuite, l'étude descriptive néerlandaise de Wevers (11) par envoi d'invitations à un échantillon de 4901 donneurs de sang identifie les caractéristiques du comportement de retour des donneurs. L'étude met en avant la possibilité de créer un engagement au début de la carrière des donneurs, par exemple en officialisant le « don de sang » sous la forme d'un « contrat social ». Selon cette hypothèse, le donneur confirme et accepte la pratique de contact avec le centre de transfusion. De ce fait, le donneur a pu prendre conscience de la possible régularité de son don, ce qui renforce sa motivation de devenir un donneur du sang fidèle.
L'étude descriptive de Godin (6) qui rassemble un échantillon de 2231 donneurs entre 18 et 70 ans comprend 2070 donneurs expérimentés et 161 nouveaux donneurs. L'objectif de cette étude est d'identifier les facteurs prédictifs auprès des nouveaux donneurs ainsi que des donneurs expérimentés. Sur la base de la TCP, un questionnaire explore trois concepts fondamentaux : l'attitude, la norme subjective et le contrôle comportemental perçu. II ressort que l'âge et l'intention sont des facteurs déterminants dans le renouvellement du don à six mois. II faut néanmoins prendre en considération que l'âge est un facteur prédictif dans la concrétisation du comportement 


\section{Interventions infirmières pour promouvoir la fidélisation des donneurs de sang : une revue rapide et réaliste de la littérature}

de don : les jeunes sont plus souvent confrontés à des situations de vie instables empêchant ainsi d'effectuer le don régulier (traduction libre, p.1610).

L'étude réalisée par Hyde (28) composée d'un échantillon de 258 étudiants, âgés entre 17 et 57 ans utilise la TCP pour comprendre le don. Parmi les 258 participants, 67 d'entre eux n'ont pas pu participer au don de sang ou d'organes. Dans cette étude, la TCP a inclus : la norme morale, l'auto-identité et l'altruisme intra-groupal afin de prédire le don. Cette dernière démontre que les attitudes communes, l'auto-efficacité et l'identité personnelle ont été les principaux contributeurs aux intentions dans les divers contextes. La norme morale, l'identité de soi et l'altruisme intra-groupal amélioraient significativement la prédiction des intentions des donneurs. Par ailleurs, la recherche suggère que les personnes qui donnent du sang auront les attitudes les plus positives et seront les plus disposées à donner des organes après leur mort et potentiellement de s'engager dans d'autres types de dons médicaux tels que le don de tissus ou de moelle osseuse.

L'étude de Chen en Chine (29), effectuée à l'aide d'un échantillon de commodité incluant 366 participants, a pour objectif de comparer deux éléments internes de la TCP : le risque perçu et la confiance, dans les centres de collecte de sang, afin de savoir s'ils ont eu une influence sur les comportements à participer au don. Cette étude démontre que les participants perçoivent un risque élevé dans le don du sang. Cependant, le risque perçu n'affecte pas le comportement envers le don du sang. Les auteurs ont soulevé que la confiance des participants dans les centres de collecte est faible et ont une influence sur l'attitude au don.

\section{Des interventions amenant à concrétiser le don}

Dans l'étude réalisée par Balegh (21) les participants sont répartis dans quatre groupes d'intervention. Cette étude expérimentale examine les effets des interventions comportementales brèves sur l'intention des non-donneurs. L'échantillon est divisé aléatoirement en quatre groupes et testent respectivement : la tension appliquée (« Applied Tension » (AT) : réduire l'anxiété pendant le don du sang), une technique de relaxation, la navigation sur le Web et un groupe témoin sans intervention. Dans les trois premiers groupes, l'intention de faire un don augmente par rapport au groupe témoin. Ceux qui ont appris l'AT ont eu une augmentation plus significative d'intentions par rapport au groupe de la navigation sur internet. Ces résultats suggèrent que des interventions simples peuvent être efficaces pour augmenter l'intention chez les non-donneurs. L'analyse de la covariance a démontré que les participants aux séances de relaxation et ceux qui ont parcouru les sites internet sur le don du sang ont augmenté leur intention de donner par rapport aux autres participants qui n'ont démontré aucun changement dans leurs intentions. Le groupe qui a participé à la relaxation, à l'AT et qui n'a pas été sur le site internet a eu une augmentation significative d'intention de faire un don par rapport aux autres participants (traduction libre, p.436).

L'essai contrôlé randomisé de Godin (30) est effectué auprès de 956 nouveaux donneurs, âgés entre 18 et 70 ans. Ces derniers ont été éligibles pour un premier don, néanmoins ils ont été temporairement exclus en raison de divers critères médicaux. L'échantillon a été divisé en deux groupes : 490 participants ont eu une intervention « d'intentions de mise en œuvre » et les 466 restants n'ont pas eu d'intervention. Le but des intentions de mise en œuvre permet lorsqu'une situation particulière se présente que la personne se sente engagée à agir selon un plan bien défini pour atteindre le comportement visé. En ce qui concerne le centre de transfusion sanguine, on peut demander aux donneurs de préciser, si cela n'est pas fait, « où ", « quand » et « comment » ils reviendront afin qu'ils puissent planifier dans leur quotidien un jour et une heure spécifique pour donner leur sang. Cette étude a eu comme objectif d'évaluer l'impact de «l'intention de mise en œuvre » sur la fidélisation du nouveau donneur. II en ressort que celle-ci a pu être une stratégie utile pour promouvoir le retour des nouveaux donneurs temporairement exclus (traduction libre, p.1653).

\section{Les interventions collaboratives entre infirmières et donneurs}

L'étude de Arage (31) faite auprès de 427 professionnels de la santé ayant comme moyenne d'âge 26 ans a pour but d'évaluer les facteurs et les pratiques de don chez les professionnels de la santé. Les résultats sont collectés par un questionnaire divisé en quatre sections : les caractéristiques sociodémographiques, les connaissances sur le don de sang, l'attitude envers le don ainsi que la pratique du don de sang. Il ressort de cette étude que le fait de travailler dans la santé n'a pas d'impact plus particulier sur les motivations à donner son sang de manière régulière. Les professionnels sont cinq fois plus susceptibles de faire un don si leurs proches ont ressenti un jour un besoin en sang, tout comme donc la population générale. De plus, l'étude met en évidence que $44,3 \%$ des soignants qui ont fait auparavant un premier don ne renouvelaient pas leur don sans motif explicable.

Selon Bani (10), différents obstacles mentionnés par les donneurs pourraient empêcher la fidélisation, tels que : la peur des aiguilles, l'évanouissement ainsi que la découverte d'une maladie. Ces différents obstacles sont connus des infirmières qui, grâce à leurs compétences et expériences, peuvent trouver différentes stratégies de mise en confiance et ainsi apaiser ces craintes qui pourraient nuire au don. En effet, ces différentes interventions établies par les infirmières permettraient de créer une relation de confiance avec les donneurs afin d'assurer leur fidélisation. Pour cela, les infirmières peuvent se baser sur l'approche centrée de la personne, le soutien émotionnel, l'échange d'informations 
pertinentes et l'écoute active. Dans l'étude de Wevers (11) ressort une autre option qui pourrait améliorer le comportement de retour du donneur qui consisterait à utiliser des « intentions de mise en œuvre » mentionnées plus haut. Cela signifie la mise en application de plans afin de faciliter la conversion des intentions en comportement (31).

\section{DISCUSSION}

Les articles synthétisés sont ici discutés au regard des trois concepts-clés de Nola Pender pour proposer des pistes de réponses à la question de recherche qui explore les interventions infirmières existantes participant à la fidélisation des donneurs de sang.

\section{Les donneurs : " caractéristiques et expériences individuelles »}

Une étude qualitative (32) réalisée auprès de 15 participants par échantillon intentionnel présente deux objectifs : l'identification et la compréhension des différentes motivations des donneurs, ainsi que l'impact de la relation entre le donneur et le professionnel de la santé. Au départ, les futurs donneurs se présentent comme bénévoles et doivent par la suite effectuer au minimum deux dons sur une période de 12 mois pour pouvoir participer à l'étude. Les résultats saillants sont que pour les donneurs, cette démarche est effectivement altruiste, car elle leur donne la possibilité de sauver des vies. De plus, les donneurs aspirent à créer un sentiment de réciprocité en envisageant qu'un jour eux-mêmes ou leurs proches puissent avoir besoin de sang. Si les donneurs font ce don, c'est avant tout pour eux-mêmes et pour leurs proches. Au-delà de ce ressenti, viennent s'ajouter des sentiments : la satisfaction, le bien-être et le bonheur d'avoir exercé une bonne action. Ces données qualitatives corroborent, expliquent et renforcent les motivations mesurées dans les articles synthétisés.

Selon Pender (18), l'individu est un être biopsychosocial qui interagit avec son environnement et laisse aussi l'environnement le transformer. Différentes interventions contextuelles simples (21), telles que les intentions de mise en œuvre, peuvent être efficaces pour augmenter l'intention des non-donneurs à donner leur sang. Les interventions testées présentées précédemment peuvent avoir un impact et ainsi participer à cibler sélectivement les différents obstacles associés au don de sang. Toutefois, selon Pender, ce nouveau comportement peut aussi être dépendant de son éducation, ses croyances et son expérience : suite à une attitude favorable au don du sang, le donneur ou le futur donneur évalue les avantages et les obstacles de cette action et par la suite s'interroge sur les moyens possibles de l'accomplissement du don. II est donc impératif pour l'infirmière de prendre en compte le ressenti éprouvé par le donneur, car plus le ressenti sera positif, plus le sentiment d'efficacité sera important.

\section{L'infirmière : « un acteur de l'environnement qui influence les personnes dans le courant de leur vie »}

Le donneur est le principal acteur du don, mais l'infirmière a un rôle indispensable dans sa participation à l'orientation des soins et services, donc dans la promotion de la santé. En effet, il ressort de l'étude de Giacomini (32), que l'infirmière apporte des informations nécessaires aux personnes susceptibles de devenir des donneurs afin de réduire leur peur et leurs croyances concernant le don du sang. Elle accompagne le donneur dans sa démarche, et ce jusqu'à ce qu'il participe activement au processus de don de manière responsable et autonome. II est important de souligner que ces mécanismes favorisent alors la fidélisation. L'infirmière intervient dans tout le déroulement du don et peut lever les divers obstacles pouvant l'empêcher comme par exemple lors du dépistage d'éléments pouvant entraver le don. Ceci grâce à différentes compétences acquises durant sa formation mais également à son expérience au sein des centres de transfusion (16,17). Le dépistage clinique lors du processus de don du sang peut paraitre comme une enquête personnelle dans l'intimité de la personne et donc peut être perçu comme intrusif. Il s'agit d'un processus primordial et impératif qui vise à acquérir principalement la bonne sécurité pendant le don. Les infirmières qui réalisent cette enquête mobilisent l'éducation, la connaissance, le conseil, l'expertise et les particularités de la personne (17). Leurs compétences visent à prévenir tout comportement pouvant être perçu comme à risque et à prévenir et à avoir des réponses fiables et sûres face aux besoins du futur donneur.

\section{| « Auto-efficacité » : les interventions infirmières}

Wevers (11) a identifié les obstacles mentionnés lors d'un refus de don. Les principaux obstacles relevés sont les contraintes de temps à $35 \%$, la préférence de reporter le don en raison de problèmes physiques généraux, bien qu'ayant le droit de faire un don à $29 \%$ et l'impossibilité d'effectuer un don en raison de critères d'exclusion médicale à $9 \%$. II est nécessaire de relever l'importance de l'absence de temps, qui constitue l'obstacle le plus fréquent pour les non-donneurs. Dans l'étude, cette barrière est comparée au concept de "l'inertie sociale ». Ce concept fait référence à la situation dans laquelle une personne a une attitude positive à l'égard d'une action mais ne prend pas les mesures pour agir pour différentes raisons. En général, les non-donneurs ont eu l'intention de participer au don mais il se peut que certains n'aient pas ressenti l'urgence d'effectuer ce don. Or, l'autoefficacité de Pender co-construite avec la personne lui permet de juger de sa capacité d'organisation et d'exécution d'un comportement et facilite la mise en œuvre du don.

Dans l'étude de Araujo (33) qui évalue le degré de satisfaction des donneurs concernant les étapes du don, les auteurs ont pu identifier une série de potentiels obstacles 


\section{Interventions infirmières pour promouvoir la fidélisation des donneurs de sang : une revue rapide et réaliste de la littérature}

à la fidélisation : le manque de clarté des soignants envers les donneurs, un manque de pertinence dans les questions médicales, des entretiens médico-infirmiers trop rapides, une banalisation de l'appréhension des soins, comme par exemple le fait de dire " ce n'est qu'une piqûre ", ou encore un apport d'informations insuffisants au sujet du don de sang. L'infirmière comprend l'impact que le don du sang peut avoir, ainsi que les questionnements qu'il engendre. Selon Pender (18), elle intervient alors selon une approche empathique, est à l'écoute, est réceptive aux inquiétudes et aux peurs des donneurs, tout en valorisant l'acte humaniste qu'ils sont prêts à faire. Ceci implique qu'elles abordent les questions en lien avec le don du sang de manière journalière. Elles sont riches de connaissances sur ce sujet et ceci doit être valorisé. L'infirmière en tant qu'experte en soins explique et met en avant la sécurité. En effet, les articles analysés démontrent l'importance de la communication entre le soignant et le donneur sur la sécurité des donneurs. En agissant de la sorte, l'infirmière s'ancre dans une posture centrée sur le donneur.

\section{《L La mise en œuvre de comportements 》 : les interventions infirmière-donneur}

Dans l'étude de Weversb (11) il est proposé la possibilité de créer un engagement au début de la carrière des donneurs, en officialisant le don de sang sous la forme d'un " contrat social ". Dans l'étude de Giacomini et al. (32), plusieurs donneurs ont mentionné l'importance des attitudes positives que les professionnels adoptent envers eux, comme par exemple : la patience, le calme, la bonne humeur et l'empathie. Ces attitudes valorisées par les donneurs permettent la diminution de l'anxiété et de la peur. De plus, il est mentionné l'importance de retenir que les donneurs désirent et ont besoin de recevoir des soins attentifs, respectueux et des informations par l'équipe soignante, que leur contribution soit valorisée. Cette interaction entre l'infirmière et les donneurs est un facteur clé pour gagner la fidélisation de ces derniers. Pour penser en termes de sensibilisation des donneurs, le modèle de Nola Pender mentionne que la mise en œuvre de ce comportement nouveau peut être favorisée par des interventions adaptées au quotidien de la personne (18).

\section{LIMITES}

La présente revue de littérature rapide et réaliste comporte certaines limites. Tout d'abord, les recherches des articles sélectionnés n'ont pas été conduites en Suisse, cependant, des articles proviennent de pays industrialisés pour lesquels certains aspects des résultats sont transférables à la Suisse : le Canada, l'Australie, et l'Italie. D'autre part, trois études sélectionnées ont été effectuées dans des pays à un stade différent de développement (Brésil et Ethiopie). Ces pays présentent probablement des différences dans la manière d'appréhender le don du sang, notamment en termes de croyances, cultures et situations socioéconomiques. Cependant, l'approvisionnement insuffisant en sang touche tous les pays cités, ce qui permet des pistes de réflexions futures au regard du cadre global de l'OMS qui vise un mouvement mondial pour le volontariat au don du sang.

\section{RECOMMANDATIONS ET PERSPECTIVES}

\section{Recommandations}

Il est important d'approfondir par des recherches qualitatives les connaissances actuelles sur les mécanismes d'engagement, les motivations et les croyances des donneurs dans le contexte suisse, puis dans le contexte genevois qui possède des particularités sociodémographiques. L'étude de Giacomini (32), précise que les professionnels de santé doivent s'appuyer sur la sécurité et sur le degré de satisfaction du donneur pendant le soin, afin de permettre leur fidélisation. Les résultats de cette revue de littérature montrent en outre que l'infirmière joue un rôle très important en ce qui concerne les donneurs de sang et leurs motivations. Elle participe aux différentes phases du processus de don du sang. Il est donc important d'intégrer ces questions à leur formation, notamment en santé publique. Ce rôle infirmier dans la promotion de la santé et la participation à la réorientation des services doit être élargi, reconnu et valorisé.

\section{Perspectives organisationnelles}

Parmi les stratégies de fidélisation du donneur de sang, il a été rapporté dans cette revue différents obstacles liés à l'organisation, comme le long délai d'attente, un environnement matériel peu agréable ainsi que des obstacles tels que des problèmes médicaux ou la peur de donner du sang. Alors que le donneur est décrit habituellement comme étant altruiste, certaines études précisent que rares sont les occasions de donner son sang sans cette motivation particulière. En Europe, afin de fidéliser les donneurs réguliers et de motiver les donneurs novices, les institutions les invitent en leur envoyant des lettres, des dépliants et font passer des campagnes de prévention à la radio. De plus, au Pays-Bas, une étude de 2009 avec un échantillon de 4901 donneurs de sang a évalué que sur une période d'un mois, $55 \%$ des donneurs de sang sont revenus pour donner du sang après avoir reçu une invitation à leur domicile (17). Au niveau organisationnel, recruter de nouveaux donneurs est un objectif ambitieux pour la plupart des CTS. L'étude de Balegh (21) suggère que les interventions testées peuvent avoir un impact et ainsi participent à cibler sélectivement certains obstacles au don de sang. Ces observations rejoignent la littérature qui démontre qu'agir aussi directement dans l'environnement du futur donneur peut avoir un impact positif sur son intention envers le don. 


\section{CONCLUSION}

Cette revue réaliste de littérature souligne l'importance des caractéristiques individuelles de chaque donneur. II est démontré par la théorie de Nola Pender, que si durant la prise en soins le donneur a un ressenti positif, il aura tendance à avoir un sentiment d'efficacité puis l'envie de revenir donner son sang. Par ailleurs, cette synthèse souligne que les donneurs peuvent créer un sentiment de réciprocité pendant le don, en présence de l'infirmière, en imaginant qu'un jour eux-mêmes puissent en avoir besoin. L'infirmière joue ce rôle dans la démarche en visant la fidélisation.

\section{DÉCLARATION DE CONFLITS D'INTÉRÊTS}

Aucun conflit d'intérêt n'est à déclarer.

\section{Références}

1. Organisation Mondiale de la Santé (OMS). Suisse [En ligne] Genève: OMS; 2016. [cité le 9 septembre 2017]. Disponible: http://www.who.int/countries/che/fr/

2. Duboz P, Lazaygues C, Boëtsch G, Chiaroni J. Donneurs de sang réguliers ou donneurs occasionnels: différences sociodémographiques et motivationnelles. Transfusion clinique et biologique. 2012 Fev;19(1):17-24.

3. Suemnig A, Konerding U, Hron G, Lubenow N, Alpen U, Hoffmann $W$, et al. Motivational factors for blood donation in first-time donors and repeat donors: a cross-sectional study in West Pomerania. Transfus Med. 2017 Dec;27(6):413-20.

4. Organisation Mondiale de la Santé (OMS). Vers 100\% de dons de sang volontaires. Cadre mondial d'action [En ligne]. Genève: OMS; 2010 [cité le 9 septembre 2017]. Disponible: https://www.who.int/ bloodsafety/publications/9789242599695.pdf?ua=1

5. Guarnaccia C, Giannone F, Falgares G, Caligaris AO, Sales-Wuillemin E. Differences in social representation of blood donation between donors and non-donors: an empirical study. Blood Transfus. 2016 Nov;14(6):487-89.

6. Godin G, Conner M, Sheeran P, Bélanger-Gravel A, Germain M. Determinants of repeated blood donation among new and experienced blood donors. Transfusion . 2007 Sep;47(9):1607-15.

7. Godin G, Germain M, Conner M, Delage G, Sheeran P. Promoting the return of lapsed blood donors: a seven-arm randomized controlled trial of the question-behavior effect. Health Psychol. 2014 Jul;33(7):646-55.

8. Myhal G, Godin G, Dubuc S. The relative efficacy of three interventions to favour return to give blood. Blood Transfus. 2017 Sep;15(5):398-404.

9. Ambroise L, Prim-Allaz I, Séville M. Attirer et fidéliser les donneurs de sang [En ligne]. Etablissement français du sang; 2010. [cité le 9 septembre 2017]. Disponible: https://halshs.archives-ouvertes. fr/halshs-00519515v2/document
10. Bani M, Strepparava M. Motivation in Italian whole blood donors and the role of commitment. Psychol Health Med. 2011 Dec;16(6):642-49.

11. Wevers A, Wigboldus D, de Kort W, Van Baaren R, Veldhuizen I. Characteristics of donors who do or do not return to give blood and barriers to their return. Blood Transfus. 2014 Jan;12(1 Suppl):37-43.

12. Aldamiz-Echevarria C, Aguirre-Garcia MS. A behavior model for blood donors and marketing strategies to retain and attract them. Rev Lat Am Enfermagen. 2014 May-Jun;22(3):467-75.

13. Transfusion CRS Suisse. Rapport annuel de transfusion CRS Suisse 2017 [En ligne]. Transfusion CRS Suisse; 2017. [cité le 9 septembre 2016]. Disponible: https://www.blutspende.ch/ $\mathrm{fr} /$ don_de_sang/actualites/rapport-annuel-de-transfusion-crssuisse

14. Hôpitaux Universitaires de Genève (HUG). Don du sang [En ligne]. Genève: HUG; 2017. [cité le 9 septembre 2017]. Disponible: http://www.hug-ge.ch/don-du-sang

15. Association des médecins du canton de Genève (AMGe). Genève vit une grave pénurie de dons du sang [En ligne]. AMGe; 2013. [cité le 9 septembre 2017]. Disponible: https://www.amge. ch/2013/01/07/geneve-vit-une-grave-penurie-de-dons-du-sang/

16. Dunkley E. Blood donation: I'm there. Br J Nurs. 2017 Jun;26(11):586.

17. Padilha ZD., Witt RR. [Nurse's competencies for clinic screening of blood donors]. Rev Bras Enferm. 2011 Mar-Apr;64(2):234-40.

18. Pender NJ. Health Promotion Model Manual [En ligne]. Ann Arbor (Michigan): University of Michigan; 2011. [cité le 9 septembre 2017]. Disponible: http://hdl.handle.net/2027.42/85350

19. Alligood MR. Nursing theorists and their work. 8th ed. Maryland Heights (Missouri); 2013. 768 p.

20. Fawcett J. Contemporary nursing knowledge: analysis and evaluation of nursing models and theories. 2nd ed. Philadelphia (Pennsylvania): F.A Davis Company; 2005. 544 p.

21. Balegh S, Marcus N, Dubuc S, Godin G, France CR, Ditto B,et al. Increasing nondonors' intention to give blood: addressing common barriers. Transfusion. 2016 Feb;56(2):433-9.

22. Pawson R, Tilley N. Realistic evaluation. London: Sage Publications; 1997. $254 \mathrm{p}$

23. Pawson R. The science of evaluation. A realist manifesto. London: Sage Publications; 2013. 240 p.

24. Saul JE, Willis CD, Bitz J, Best A. A time-responsive tool for informing policy making: rapid realist review. Implement Sci. 2013 Sep;8:103.

25. Pawson R. Evidence-Based Policy: A Realist Perspective. olicy: A Realist Perspective. Thousand Oaks (California): Sage; 2006. $209 p$.

26. Polit DF, Beck CT. Nursing Research. Generating and Assessing Evidence for Nursing Practice. 10th ed. Philadelphia (Pennsylvania): Lippincott Williams and Wilkins; 2016. 768 p.

27. Ajzen I. The theory of planned behavior. Organ Behav Hum Decis Process. 1991 Dec;50(2):179-211. 


\section{Interventions infirmières pour promouvoir la fidélisation des donneurs de sang : une revue rapide et réaliste de la littérature}

28. Hyde MK, Knowles SR, White KM. Donating blood and organs: using an extended theory of planned behavior perspective to identify similarities and differences in individual motivations to donate. Health Educ Res. 2013 Dec;28(6):1092-10.

29. Chen L. Applying the extended theory of planned behaviour to predict Chinese people's non-remunerated blood donation intention and behaviour: the roles of perceived risk and trust in blood collection agencies. Asian J Soc Psychol. 2017 Jul-Oct;20(3-4):221-31.

30. Godin G, Amireault S, Vézina-Im L, Sheeran P, Conner M, Germain $\mathrm{M}$, et al. Implementation intentions intervention among temporarily deferred novice blood donors. Transfusion 2013 Aug;53(8):1653-60.
31. Arage G, Ibrahim S, Adimasu E. Blood donation practice and its associated factors among health professionals of University of Gondar Hospital, Northwest Ethiopia: a cross sectional study. BMC Res Notes. 2017 Jul;10(1):294.

32. Giacomini L, Danilo W, Lunardi F. Estratègias para fidelização de doadores de sangue voluntários e habituais. Acta Paul Enferm 2010;23(1):65-72

33. Araujo F, Feliciano K, Mendes MF. [Acceptance by blood donors of the public blood bank in Recife, Brazil]. Cien Saude Colet. 2011 Dec;16(12):4823-32. 